\title{
ACTUALIZACIÓN DEL PAPEL DE LAS ANALOGÍAS VERBALES Y SU CAPACIDAD PREDICTIVA SOBRE LA PRUEBA FINAL DE LA ESO
}

\author{
Gregorio Pérez Bonet \\ goyo@cesdonbosco.bom \\ "Universidad CES Don Bosco" adscrita a la Universidad Complutense de Madrid, España.
}

Fecha de Recepción: 13 Agosto 2018

Fecha de Admisión: 1 Octubre 2018

\section{RESUMEN}

Con el presente trabajo se propone un formato nuevo de evaluación mediante analogías verbales con la prueba AN2.0. Se ha pilotado la prueba con alumnado de $4^{\circ}$ de la $E S O, N=74$. Se obtiene un de cronbach de .85. Así mismo muestra buenas correlaciones con DAT RV y DAT RN, así como con la prueba de final de etapa de la ESO de la Comunidad de Madrid, convirtiéndose en el mejor predictor de la misma. Se confirma, así, el gran peso asociativo de las analogías verbales con el rendimiento escolar incluso con variantes en el formato de respuesta, abriéndose de esta manera un nuevo campo a explorar.

Palabras clave: analogías verbales; razonamiento; rendimiento escolar; evaluación final ESO

\section{ABSTRACT \\ UPDATING OF THE ROLE OF VERBAL ANALOGIES AND THEIR PREDICTIVE CAPACITY ON FINAL ESO EXAM}

With the present work proposes a new format of assessment using verbal analogies with the test AN2.0. It has been piloted the test with students of $4^{\circ} \mathrm{ESO}, \mathrm{N}=74$. The test gets Cronbach's Alpha of .85. At the same time shows good correlation with DAT RV and DAT RN, as well as with the final exam of ESO ( Community of Madrid), becoming the best predictor of the califications in the exam. It is confirmed, the great role of the verbal analogies in the scholar achievement even with changes in the format of response, opening a new field to explore.

Keywords: verbal analogies; reasoning; school achievement;final exam ESO

\section{INTRODUCCIÓN}

Dentro de los complejos procesos sociales que generan innumerables y rápidos cambios en los ámbitos laboral y educativo, la orientación se perfila como una herramienta obligada.

Dicha orientación se entiende habitualmente como un abanico de actividades que capacita a 
Ios ciudadanos en cualquier etapa de su vida a: 1) analizar sus competencias e intereses, 2) adoptar decisiones de formación y empleo y 3) gestionar su trayectoria vital en cuanto al aprendizaje , el empleo y otras cuestiones en las que se adquieren o utilizan competencias (Consejo de la Unión Europea, 2004).

En nuestro contexto español, los programas de orientación destinados a la ESO (Educación Secundaria Obligatoria) responden a una doble perspectiva desde el eje temporal. Por un lado, se desarrolla un enfoque continuado vertebrado principalmente por la acción tutorial. Y por otro, se aplica un enfoque más puntual e intensivo con atención especial a los momentos en los que los alumnos tienen que elegir. Dicha elección puede vincularse, 0 bien a materias optativas, ramas 0 especialidades 0 relacionarse con el acceso al mundo laboral. La orientación en $4^{0}$ de la ESO se antoja relevante, pues en este curso se culmina la educación obligatoria dando lugar a diversas posibilidades siempre que se titule: mundo laboral, formación profesional grado medio o bachillerato en alguna de las modalidades previstas por la ley.

Desde la perspectiva anterior resulta fundamental recurrir al análisis tanto de factores individuales y factores contextuales más genéricos para adoptar las medidas oportunas para una intervención preventiva (Do Ceu y Rodríguez, 2003, Ecclestone y Pryor, 2003, Sebastián, 2003, Sarmiento y Ocampo, 2004) así como una orientación eficaz.

A pesar del profundo viraje en torno a la multidimensionalidad del rendimiento escolar incorporando variables, tanto cognitivas como de personalidad, que a su vez están influenciadas por aspectos individuales, educativos y familiares (Jiménez y López Zafra, 2009), la inteligencia como variable está más que demostrada por la mayoría de autores (Fajardo Bullón, F y cols, 2017). Aunque ello no implica la ausencia de limitaciones y controversias. Las pruebas cognitivas han sido y continúan siendo buenos predictores del rendimiento académico (Ridgell \& Lounsbury, 2004; Dollinger \& Orf, 1991) en todas las etapas educativas. No obstante, el peso de las mismas, en el propio rendimiento escolar parece ir decreciendo a medida que vamos avanzando en la pirámide de niveles educativos (Descals y Rivas, 2002, Almeida, 1988, Ribeiro, 1998).

Es decir, que en la educación primaria y secundaria existiría una relación más consistente que en la etapa universitaria. Lo que los estudios apuntan en la relación entre medidas de la inteligencia y el rendimiento académico, en términos correlacionales, se situarían en torno a 0.5 (Descals y Rivas, 2002).

Dentro del amplio espectro de pruebas intelectuales, las pruebas de razonamiento lógico ya sean con soporte verbal, numérico o viso-espacial han tenido gran acogida por su valor predictivo del rendimiento escolar en la tradición de orientadores escolares. Y dentro de estas, aquellas con soporte verbal han indicado una mayor asociación con el rendimiento general del alumno que otras de razonamiento 0 de inteligencia general (Cascón, 2000). De este modo, se justificaría la mayor extensión de pruebas de aptitudes diferenciales (razonamiento verbal y matemático principalmente) como alternativa a los test de factor " $g$ " o de C.I. en la consulta de orientación vocacional de adolescentes y adultos (Almeida et al, 2008). Incluso, eliminando el efecto del factor " $g$ " de inteligencia se siguen encontrando relaciones consistentes con el rendimiento escolar (Coyley, 2014, Coyley \& Pillow, 2008) de estas aptitudes. Ello es así, probablemente, para el caso del razonamiento verbal, debido a que estaría incidiendo en todas las áreas del currículo, a pesar de ser un factor susceptible de influencias socioculturales y familiares (Pérez Sánchez \& Castejón Costa, 1997). Las investigaciones han encontrado de forma recurrente que el factor comprensión verbal o Factor $V$ estaría implicado en tareas de índole diversa como comprensión de frases desordenadas, selección de sinónimos y antónimos, vocabulario, analogías verbales o razonamiento verbal (Yela, 1996), aspectos estrechamente relacionados con las competencias escolares (Robles, y Vázquez González, 2014) y la denominada inteligencia cristalizada. 
En cualquier caso, parece que el factor " $g$ " no estaría del todo ajeno en su influencia hacia las propias aptitudes, como es el caso del razonamiento verbal. Desde la propuesta de Catell-HornCarroll consistente en tres estratos, encontraríamos el factor " $g$ " en lo alto del modelo extendiendo su impacto a todas las capas inferiores en mayor o menor gado. En un segundo nivel estarían una decena de factores asociados a procesos cognitivos (velocidad de procesamiento, capacidad de almacenaje, evocación de información...). En el estrato más bajo se hallarían factores más específicos (vocabulario, numérico, visoespacial...) ligado al concepto tradicional de aptitud diferencial (Marrs, 2011).

Sería en esta capa donde encontraríamos vínculos con el razonamiento verbal de forma operativa, pero sin olvidar las relaciones con los estratos superiores o dicho de otra forma, sus correlaciones con el factor "g" ( Kuncel, Hezbett \& Ones, 2004).

Según la evidencia de las últimas décadas, parece que la forma más idónea de evaluar el razonamiento verbal es a través de analogías verbales (Kuncel, et al , 2004 Stenberg y Berg, 1992). De hecho, en el contexto de la orientación académica y profesional en USA se emplean de forma frecuente el "Miller Analogies Test" (MAT), el "Graduate Record Exam" (GRE) 0 el "Scholastic Aptitud Test" (SAT). Dichas pruebas no han estado exentas de críticas, principalmente vinculados a la posible segregación de minorías étnicas, lo que en algunos casos supuso la reformularon de las analogías verbales y en otros su eliminación (Geiser, 2016). En nuestro entorno, quizás el test de analogías verbales más utilizado es el DAT-RV, de la prueba DAT-5. Una posible explicación de tan extendido uso estriba, como sugeríamos anteriormente, en que este tipo de pruebas pueden incluir conocimientos de una amplia gama de dominios que están representados en los conceptos a relacionar abarcando distintas áreas; matemáticas, lengua, ciencias, música... De esta forma, podemos aumentar los coeficientes de correlación cuando los procesos cognitivos y el contenido de los ítems de las pruebas tienen mayor afinidad con las disciplinas curriculares (Floy et al, 2003, Mc Grew y Evans, 2002).

Sin embargo, y a pesar del nutrido cuerpo de evidencias, las analogías verbales, resultan para algunos, un asunto ya obsoleto. En Junio de 2006 un artículo del "New York Times" titulaba "Forget Analogies, Reason More, Memorize less". El cierto cansancio de estas pruebas que pueden penalizar a aquellos con menos exposición cultural puede estar en la base de este supuesto desencanto. Por el contrario, y para añadir un ejemplo más, en el metanálisis de Kuncel, et al (2004) sobre el test de analogías por excelencia, el MAT, concluían que esta prueba resultaba una potente herramienta de habilidad cognitiva general ya que requieren razonamiento con vocabulario y conocimiento de varios dominios y que existía una fuerte asociación con el rendimiento académico y ajuste laboral.

Las pruebas de analogías verbales consisten habitualmente en un par de palabras vinculadas por una relación que se ha de comparar con otro que tienen una relación análoga. Aunque los formatos de presentación de este tipo de pruebas pueden variar, la forma más común consiste en presentar tres palabras y elegir la cuarta de entre una opción múltiple. Otra de las formas también frecuentes es presentar un par de palabras y escoger el par de palabras que presenta la misma relación de entre una opción múltiple (Attorresi, Panio, Fernández, Liperace \& Caissials, 1993).

Esta investigación trata de responder a una pregunta general ¿Están agotados los modelos de pruebas basadas en analogías verbales en la orientación escolar ? Para ello, en este trabajo, que pertenece una investigación más amplia, se estudian las relaciones de las analogías verbales y su capacidad predictiva de la prueba final de la ESO, frente a otras pruebas de inteligencia general o lógico matemáticas, sugiriendo un nuevo formato de analogías verbales. 


\section{OBJETIVOS}

Tras la revisión de la literatura científica vinculada al tema de nuestro estudio, nos proponemos los siguientes objetivos:

Realizar el pilotaje de una prueba de analogías verbales como herramienta de orientación para $4^{\circ}$ de la ESO (AN2.0).

Comprobar el nivel de asociación entre las pruebas de razonamiento verbal mediante analogías verbales, el razonamiento matemático, la inteligencia general, el rendimiento escolar y la prueba final de la ESO.

Establecer el valor predictivo de las variables anteriores con la prueba final de etapa de la Comunidad de Madrid.

\section{MÉTODO}

El diseño de la investigación es de tipo descriptivo y correlacional, ya que se pretendió conocer los niveles de los participantes en las variables medidas, y estudiar las relaciones de las mismas así como el valor predictivo de alguna de ellas.

\section{PARTICIPANTES}

La muestra es no probabilística y de tipo incidental, ya que formaron parte del estudio todos los alumnos de 4ํㅡㄹ la ESO de dos colegios concertados del distrito "Salamanca" de Madrid dentro del programa ordinario de orientación $(N=78)$. Ambos centros pertenecen a la misma congregación religiosa y apenas distan un kilómetro entre ellos. La muestra final la han constituido 74 alumnos de los 4 grupos de $4^{\circ}$ de la ESO (dos por cada colegio). El alumnado se distribuye entre la modalidad de académicas ( $N=52)$ y en la de aplicadas ( $N=22)$. La distribución por géneros es de 34 alumnos $(45,9 \%)$ y 40 alumnas (54,1\%). La edad media es de 15 años y 9 meses.

\section{INSTRUMENTOS}

\section{Pruebas de aptitudes e inteligencia general}

Para la evaluación de aptitudes se han utilizado dos pruebas: el test de razonamiento verbal DATRV de la prueba DAT-5 (Bennet, Seashore \& Wesman, 2000) y el test de razonamiento matemático DAT-RN de la misma batería. Ambas pruebas constituyen unos buenos indicadores del rendimiento académico con coeficientes de correlación promedio de 0,45 en numerosos estudios realizados tanto en USA como en Iberoamérica (Pérez, Cupani \& Ayllón, 2005). Los coeficientes de fiabilidad ( de cronbach) de la batería según los autores oscilan entre 0,75 y 0,92 para los ocho subtest, por lo que se consideran adecuados.

Se ha completado la evaluación con la prueba de inteligencia general BLS IV (Bonnardel, 1970). Dicha prueba está desprovista de contenido verbal y sus elementos se presentan con dificultad creciente. Es una prueba rápida de 10 minutos de aplicación. En nuestra muestra, el coeficiente de fiabilidad muestra un de cronbach de 0,78 lo que resulta adecuado para nuestros propósitos.

Por otro lado, se ha pilotado una prueba de analogías verbales diseñada por nuestro departamento de orientación, el AN2.0. La prueba final consta de 30 items, una vez eliminados aquellos que no arrojaban una buena consistencia interna.

El tiempo de realización es de 30 minutos. El de cronbach final es de 0,85 lo que supone una alta fiabilidad según los parámetros de George \& Mallery (2011), aunque provisional dado el tamaño muestral. Los items no están ordenados por dificultad. A diferencia de las pruebas clásicas de analogías, en esta prueba se tiene que escribir la palabra que completa la analogía. Para ello, se ofrece como pistas tantos guiones como caracteres tiene la palabra que buscamos. A continuación mostramos ejemplos con distintos niveles de dificultad en la prueba AN2.0: 


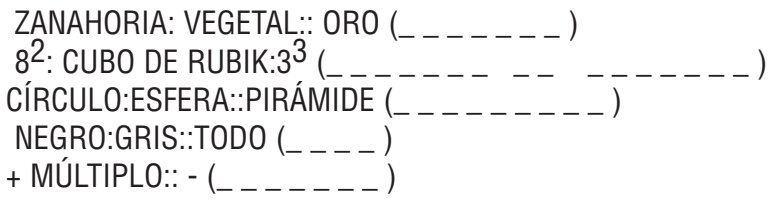

Se han tomado cada una de las respuestas como variables dicotómicas dando el valor 1 si la respuesta es correcta y 0 si es incorrecta, siguiendo otros modelos similares (Galibert, Aguerri, Pano , Luzzia y Attorresi, 2005)

\section{Calificaciones de la $2^{\text {a }}$ evaluación}

El rendimiento escolar se considera uno de los elementos fundamentales para medir el funcionamiento idóneo del ámbito educativo (Marchesi y Martín, 1999). Para ello, el indicador que resulta más fiable y válido de medición del rendimiento escolar continúan siendo las calificaciones escolares a pesar de sus críticas a la subjetividad (Córdoba, García luengo, Vizuete y Feu, 2011). En nuestro estudio hemos escogido las notas medias de las asignaturas: Matemáticas (académicas 0 aplicadas) Lengua y Literatura, Inglés y Geografía e Historia de la $2^{\text {a }}$ evaluación. Se han escogido estas materias por ser las mismas que en la prueba final de etapa de la ESO para comprobar su asociación. Se ha escogido la 2ª evaluación por ser la más cercana temporalmente con la prueba final de la ESO.

\section{Prueba final de etapa ESO}

La prueba final de la Educación Secundaria Obligatoria se constituye en tres ámbitos: competencia lingüística, que se centrará en la comprensión y expresión escrita en lengua española y en inglés, competencia matemática que implicará la resolución de problemas relacionados con la vida cotidiana y el ámbito de competencia social y cívica que se realizará a través del conocimiento y razonamiento sobre contenidos de Geografía e Historia (BOCM, 2018). Los resultados de la prueba se expresan en 6 niveles de competencia. No obstante, para nuestra investigación hemos utilizado las puntuaciones brutas de cada examen, en vez de los niveles del 1 al 6 de cara a un análisis de datos más afinado.

\section{PROCEDIMIENTO}

Dentro de las actividades propias del departamento de orientación de ambos centros, el propio autor del trabajo aplicó durante el mes de febrero del curso 2017-28 las pruebas de razonamiento verbal (DAT RV) y de razonamiento lógico matemático (DAT RN), el test de inteligencia general BLS IV, así como la prueba diseñada AN2.0. Tanto las familias como los alumnos fueron informados y se obtuvieron los permisos pertinentes a la evaluación.

En el mes de Abril del curso 2017-18 se aplicaron las pruebas de la Comunidad de Madrid durante dos días (24 y 25 de abril). A pesar de que las pruebas no eran vinculantes, se motivó al alumnado a implicarse en las mismas. En Junio se recibieron los resultados de las pruebas de la Comunidad de Madrid.

\section{ANÁLISIS DE DATOS}

Para el análisis de datos se ha utilizado el paquete estadístico SPSS 22.

En una primera estrategia descriptiva se presentan las medias y desviaciones estándar de las variables incluidas en la investigación (Tabla 1). Los índices de kurtosis y asimetría de todas la variables están comprendidos entre -1 y 1 lo que permite aplicar técnicas paramétricas (George \& Mallery, 2001). 
Respecto a la prueba a pilotar AN2.0 la media es de 11,2 sobre 30 posibles aciertos. Se comprueba que a pesar de disponer de 30 minutos el techo posible de la prueba es alto.

Con relación a las demás pruebas, (DAT-RV, DAT-RN y BLS IV) las puntuaciones medias se encuentran dentro del rango esperado para la edad, con una ligera ventaja para la prueba de razonamiento matemático frente a la de razonamiento verbal. La media de calificaciones de las cuatro asignaturas valoradas en la $2^{2}$ evaluación, y que se corresponden con las cuatro medidas en la prueba final de la Comunidad de Madrid, arroja un resultado de 5,49 sobre 10. Este dato es presumiblemente inferior al obtenido en la prueba final de la Comunidad de Madrid que es de 538,21, y que estaría aproximadamente en un nivel 4 cercano al nivel 5 de competencia (dentro de los 6 niveles establecidos).

\section{Tabla 1 Estadísticos descriptivos de las variables}

\begin{tabular}{|c|c|c|c|c|c|}
\hline & $\mathrm{N}$ & Mínimo & Máximo & Media & $\begin{array}{l}\text { Desviación } \\
\text { estándar }\end{array}$ \\
\hline AN2.0 & 74 &, 00 & 25,00 & 11,2432 & 5,52974 \\
\hline BLSIV & 74 & 1,00 & 16,00 & 10,4259 & 3,77980 \\
\hline DAT RN & 74 & 8,00 & 30,00 & 16,9412 & 5,31972 \\
\hline DAT RV & 74 & 1,00 & 39,00 & 14,7593 & 11,26178 \\
\hline MEDREND2 ${ }^{\mathrm{a}}$ & 74 & 2,00 & 9,50 & 5,4953 & 1,89530 \\
\hline MEDMAD & 74 & 400,25 & 690,00 & 538,2143 & 72,18469 \\
\hline $\mathrm{N}$ válido (por lista) & 74 & & & & \\
\hline
\end{tabular}

AN2.0. Prueba de analogías verbales a pilotar

DAT RV Prueba de Razonamiento Verbal del DAT-5

BLS IV Prueba de inteligencia general

DAT RN Prueba de razonamiento numérico del DAT-5

MEDRED2 ${ }^{\mathrm{a}}$ Media de las calificaciones de Lengua, Inglés, Matemáticas y Geografía e Historia de la $2^{\mathrm{a}}$ evaluación

MEDMAD Media de las calificaciones de la Prueba final de la ESO (Lengua, Inglés, Matemáticas, Social y Cívica)

En ninguna de las variables descritas aparecen diferencias significativas tras la aplicación de $t$ de student para muestras independientes según la variable género. ( $p>, 05)$, lo que indica que para esta muestra no existe sesgo de género en ninguna de las pruebas.

Respecto a la prueba de la Comunidad de Madrid presentamos los resultados de las cuatro áreas valoradas (Tabla 2).

Tabla 2 Estadísticos descriptivos de la prueba final de la ESO

\begin{tabular}{|l|l|l|l|l|l|l|l|}
\hline & $\mathrm{N}$ & Rango & Mínimo & Máximo & Media & Desviación estándar & Varianza \\
\hline LENGMA & 74 & 405,00 & 311,00 & 716,00 & 525,2222 & 89,51173 & 8012,349 \\
D & 74 & 381,00 & 346,00 & 727,00 & 522,9444 & 101,15644 & 10232,62 \\
INGMAD & 74 & 430,00 & 259,00 & 689,00 & 525,5429 & 73,76407 & 5441,138 \\
MATMAD & 74 & 289,00 & 437,00 & 726,00 & 580,0833 & 77,99684 & 6083,507 \\
SOCYCIV \\
$\begin{array}{l}\text { N válido } \\
\text { (por lista) }\end{array}$ & 74 & & & & & & \\
\hline
\end{tabular}

LENGAMD= Calificación de la prueba de Lengua

INGMAD $=$ Calificación de la prueba de Inglés

MATMAD $=$ Calificación de matemáticas (Académicas o Aplicadas)

SOCYCIV= Calificación del área Social y Cívica 
Las puntuaciones medias de las cuatro áreas de la prueba de la Comunidad de Madrid son bastante homogéneas; principalmente son muy parecidas entre Lengua con 525,2 (nivel 4), Inglés con 522,94 (Nivel 4) y Matemáticas con 525,54 (Nivel 4). En el área social y cívica es en la que se obtiene la media más alta con 580,08 (Nivel 5).

Seguidamente, se realiza una exploración de los datos a partir de un análisis correlacional bivariado ( $r$ de Pearson) de las variables incluidas con el objetivo de comprobar el nivel de asociación entre ellas.

En la matriz de correlaciones (Tabla 3) encontramos una buena capacidad asociativa de la prueba diseñada AN2.0 con las pruebas de razonamiento lógico, tanto con las de carácter verbal (AN2.0 con DAT VR $=, 780^{\star *}$ ) como las de razonamiento lógico matemático (AN2.0 con DAT RN $=, 795^{\star *}$ ). Todas estas puntuaciones indican una buena capacidad para la valoración de procesos lógicos de la prueba a pilotar. Finalmente, encontramos también una correlación prácticamente alta (cercana a $0,7)$ con la inteligencia general (AN2.0 con BLS IV $=, 697^{* *}$ ) aunque en menor medida que las pruebas de razonamiento anteriores.

Se encuentra una asociación moderada de AN2.0 (.599**) respecto a la media de las calificaciones de la 2aㅡ evaluación (Matemáticas, lengua, Inglés y Geografía e Historia) de los cuatro grupos de $4^{0}$ de la ESO. Sin embargo, respecto a la media de las cuatro pruebas de la prueba final de la Comunidad de Madrid (MEDMAD), aparece una asociación más alta $\left(, 781^{* *}\right)$ con la prueba AN2.0. No obstante, de forma similar, se obtienen una alta asociación entre la prueba final (MEDMAD) y la otra prueba que utiliza analogías verbales DAT-VR $\left(, 758^{* *}\right)$. La relación de la inteligencia general con el rendimiento, tanto de la 2ª evaluación como de la prueba final de la ESO es moderada; ,465** y, $481^{* *}$ respectivamente. La prueba de razonamiento matemático (DAT-RN) obtiene, de forma similar, una asociación moderada con el rendimiento de la $2^{\text {a }}$ evaluación $\left(, 429^{* \star}\right)$ y una, sensiblemente mayor, con el rendimiento medio de la prueba final de la ESO $\left(, 645^{\star *}\right)$.

Se establece, de esta forma, que las pruebas de analogías verbales tienen mayor relación con el rendimiento dentro de las variables de nuestra investigación, tanto respecto al rendimiento de la $2^{0}$ evaluación como para la prueba final de la ESO. Por otro lado, se encuentra una relación consistente entre lo medido en la $2^{\underline{a}}$ evaluación y la propia prueba final de la ESO $\left(, 709^{\star *}\right)$, sugiriendo una similar valoración de competencias académicas.

Tabla 3. Correlaciones bivariadas ( $r$ de Pearson) entre las variables.

\begin{tabular}{|l|l|l|l|l|l|l|}
\hline & $(1)$ & $(2)$ & $(3)$ & $(4)$ & $(5)$ & $(6)$ \\
\hline AN2.0 (1) & &, $780^{* *}$ &, $697^{* *}$ &, $795^{* *}$ &, $599^{* *}$ &, $781^{* *}$ \\
\hline DAT RV(2) & & &, $602^{* *}$ &, $678^{* *}$ &, $644^{* *}$ &, $758^{* *}$ \\
\hline BLSIV (3) & & & &, $589^{* *}$ &, $465^{* *}$ &, $481^{* *}$ \\
\hline DAT RN(4) & & & & &, $429^{*}$ &, $645^{* *}$ \\
\hline MEDRE2 (5) & & & & & &, $709^{* *}$ \\
\hline MEDMAD (6) & & & & & & \\
\hline
\end{tabular}

AN2.0. Prueba de analogías verbales a pilotar

DAT RV Prueba de Razonamiento Verbal del DAT-5

BLS IV Prueba de inteligencia general

DAT RN Prueba de razonamiento numérico del DAT-5

MEDRED2 ${ }^{\mathrm{a}}$ Media de las calificaciones de Lengua, Inglés, Matemáticas y Geografía e Historia de la $2^{\mathrm{a}}$ evaluación MEDMAD Media de las calificaciones de la Prueba final de la ESO (Lengua, Inglés, Matemáticas, Social y Cívica)

Finalmente, se ha incluido un análisis de regresión múltiple multivariado para establecer el valor predictivo de las variables incluidas. Se ha utilizado como variable dependiente la calificación media final de la prueba de la Comunidad de Madrid (MEDMAD). Las demás variables se han incluido como 
independientes. Se ha llevado un análisis por pasos sucesivos. En él encontramos que con dos de las variables (puntuación en el AN2.0 y la nota media de calificaciones de la $2^{0}$ evaluación) se puede predecir el $83 \%$ de la variabilidad de la nota media de la prueba final de etapa de la ESO de la Comunidad de Madrid según el segundo modelo, Y el 70\% de la misma con tan solo la puntuación de la prueba AN2.0, excluyendo todas las demás variables, según el primer modelo

Tabla 4: Resumen de modelos

\begin{tabular}{|l|l|l|l|l|}
\hline Modelo & $\mathrm{R}$ & R cuadrado & $\begin{array}{l}\mathrm{R} \text { cuadrado } \\
\text { ajustado }\end{array}$ & $\begin{array}{l}\text { Error estándar } \\
\text { de la estimación }\end{array}$ \\
\hline 1 &, $844^{\mathrm{a}}$ &, 712 &, 702 & 39,81605 \\
2 &, $920^{\mathrm{b}}$ &, 846 &, 835 & 29,62974 \\
\hline
\end{tabular}

a. Predictores: (Constante), AN2.0

b. Predictores: (Constante), AN2.0,MEDRED2 ${ }^{\mathrm{a}}$

Tabla 5: Variables excluidas de los modelos

\begin{tabular}{|c|c|c|c|c|c|c|}
\hline \multirow{2}{*}{\multicolumn{2}{|c|}{ Modelo }} & \multirow[b]{2}{*}{ En beta } & \multirow[b]{2}{*}{$\mathrm{t}$} & \multirow[b]{2}{*}{ Sig. } & \multirow{2}{*}{$\begin{array}{l}\text { Correlación } \\
\text { parcial }\end{array}$} & \multirow{2}{*}{$\begin{array}{ll}\begin{array}{l}\text { Estadísticas de } \\
\text { colinealidad }\end{array} \\
\text { Tolerancia }\end{array}$} \\
\hline & & & & & & \\
\hline \multirow[t]{4}{*}{1} & BLS IV &,$- 093^{b}$ &,- 642 &, 526 &,- 118 & ,464 \\
\hline & DAT RV &, $366^{\mathrm{b}}$ & 2,456 &, 020 & ,415 & ,370 \\
\hline & DAT RN &, $052^{\mathrm{b}}$ &, 325 &, 747 &, 060 & ,384 \\
\hline & MEDRED $^{\circ}$ &, $452^{\mathrm{b}}$ & 5,017 & ,000 & ,682 & ,656 \\
\hline \multirow[t]{3}{*}{2} & BLSIV &,$- 089^{\mathrm{c}}$ &,- 828 & ,415 &,- 155 & ,464 \\
\hline & DAT RV &, $211^{\mathrm{c}}$ & 1,740 & ,093 & ,312 & ,339 \\
\hline & DAT RN &, $201^{\mathrm{c}}$ & 1,717 & 097 & ,309 & ,363 \\
\hline
\end{tabular}

a. Variable dependiente: MEDMAD

b. Predictores en el modelo: (Constante), AN2.0

c. Predictores en el modelo: (Constante), AN2.0, MEDRED2 ${ }^{\mathrm{a}}$

Los datos revelan, de manera consistente, que si tuviéramos que escoger únicamente una prueba de las dispuestas en el presente trabajo, elegiríamos la prueba piloto AN2.0, incluso antes que las propias notas medias de la evaluación, como predictor de la nota media en la prueba final de la ESO.

\section{DISCUSIÓN Y CONCLUSIONES}

Siguiendo el orden de los objetivos enunciados previamente podemos señalar que el pilotaje de la prueba AN2.0 con una muestra de 74 alumnos se ha realizado con razonable éxito. La prueba presenta una alta consistencia interna (.85). Respecto a la validez de contenido, el borrador pasó por una valoración inicial de profesores de psicología de dos universidades para valorar en qué medida evaluaba el razonamiento verbal. Con relación a la validez de constructo consideramos, provisionalmente, que la prueba tienen una consistente validez dadas las correlaciones con pruebas ya baremadas (AN0.2 con DAT VR=,780**). Sorprendentemente, también tiene una buena capacidad de asociación con pruebas de razonamiento lógico matemático (AN2.0 con DAT RN =,795**) y razonable con la inteligencia general (AN2.0 con BLS IV=,697**).

Los resultados, en conjunto, avalan el valor predictivo del rendimiento escolar de las pruebas de analogías verbales y son consistentes con la investigación previa. En este caso el DAT-RV, prueba ya ampliamente validada confirma su capacidad y el AN2.0 arroja también un alto nivel de predic- 
ción. De hecho, y en mayor medida que las propias calificaciones de la 2ª evaluación, el AN2.0 explica el $70 \%$ de la varianza en la nota media de la prueba final de la Comunidad de Madrid, respecto a las demás variables, convirtiéndose, para esta muestra, en la variable con mayor peso predictivo. Respecto a la prueba final de la ESO, consideramos que puede aportar información relevante, y potencialmente, con una mayor fiabilidad que las propias calificaciones escolares de las evaluaciones ordinarias. De esta manera, se pueden detectar casos de riesgo académico, sino se ha realizado antes, con presumible mayor fiabilidad que incluso el rendimiento académico de las evaluaciones ordinarias previas.

Entre las limitaciones del presente trabajo podemos señalar que hace falta aumentar la muestra de aplicación del AN2.0 para poder estudiar la estructura factorial más claramente y confirmar su validez. A pesar del tiempo de 30 minutos, para la prueba AN2.0 se puede considerar la posibilidad de acortar los tiempos con una prueba con menos ítems.

Por otro lado, consideramos relevante poder extender la prueba a otros contextos socioculturales para comprobar su comportamiento transcultural, tanto dentro de España como en otros países hispano hablantes. Además, consideramos que la prueba podría medir en menor medida otros rasgos relevantes como pensamiento divergente y flexibilidad cognitiva. Por ello animamos a otros investigadores y orientadores a colaborar en la validación de la prueba, pudiendo utilizarla como complemento para la orientación vocacional en $4^{0}$ de la ESO o curso equivalente.

Finalmente, podemos contestar provisionalmente, que las pruebas de analogías verbales no están agotadas. Esta investigación sugiere nuevas líneas de investigación en los formatos de respuesta mejorando, presumiblemente, su poder predictivo.

\section{BIBLIOGRAFÍA}

Almeida,L., Guisande, M.A., Primi, R. y Lemos, G. (2008) Contribuciones del factor general y de los factores específicos en la relación entre inteligencia y rendimiento escolar. European Journal of Education and Psychology, 1 (3), 5-16

Almeida, L.S. (1988). 0 raciocíno diferencial dos jovens: Avaliação, desenvolvimento e diferenciação, Porto: Instituto Nacional de Investigação Científica.

Attorresi, H., Pano, C., Fernández Liporace, M. \& Cayssials, A (1993). Evaluación de la habilidad para identificar y discriminar relaciones. Anuario de Investigaciones, 3, 27 - 34.

Bennett, G. K., Seashore, H. G., \& Wesman, A. G. (2000). Test de Aptitudes Diferenciales (DAT-5). Manual. Madrid: TEA Ediciones.

Boletín Oficial de la Comunidad de Madrid (2018) Otras disposiciones sobre la Evaluación final de $4^{\circ}$ de la ESO del 27 de febrero de 2018.,№ 49 pp 52-59.

Bonnardel,R. (1970). BLS-IV.Test de Inteligencia general. Madrid: MEPSA

Cascón,I. (2000). Análisis de las calificaciones escolares como criterio de rendimiento académico. En red recuperado en :http:(//www3.usal.es/inico/investigación/jornadas/ ornada2/comunc/cl7.html7ku

Consejo de la Unión Europea (2004). Proyecto de resolución del Consejo y de los representantes de los Estados miembros reunidos en el seno del Consejo sobre el fortalecimiento de las políticas, sistemas y prácticas en materia de orientación permanente en Europa. (18 de mayo de 2004),8448/4 EDUC 890 SOC1790 (Recuperado el 8 de marzo de 2018, de http://ec.europa.eu/education/lifelong-learning-policy/doc/guidance/resolution2004_es.pdf

Córdoba Caro, L., García Preciado, V., Luengo Pérez, L., Vizuete Carrizosa, M., \& Feu Molina, S. (2011). Determinantes socioculturales: su relación con el rendimiento académico en alumnos de Enseñanza Secundaria Obligatoria. Revista De Investigación Educativa, 29(1), 83-96. 
Coyle, T.R.; Pillow, D.R. (2008) SAT and ACT predict college GPA after removing g. Intelligence , 36 (6) 719-729.

Coyle,T.R. (2014) Predicitive validity of non "g" residuals of test: More than g. Intelligence, 2 (1), 21-25.

Descals, A y Rivas, F.(2002). Capacidades intelectuales y rendimiento escolar de estudiantes de secundaria: Constatación de una limitada relación. Revista Galego-portuguesa de psicoloxia e educación, 6 , 203-214.

Do Ceu, M. y Rodríguez, M.L. (2003). Guidance Theory and practice: the status of career exploration. British Journal of Guidance and Counselling, 31 (2), 189-207.

Dollinger, S. J., \& Orf, L. A. (1991). Personality and performance in "personality": Conscientiousness and openness. Journal of Research in Personality, 25 (3), 276-284.

Ecclestone, K. y Pryor, J. (2003) 'Learning careers' or 'Assessment Careers'? The impact of assessment systems on learning. British Educational Research Journal, 29 (4), 471-488.

Fajardo Bullón, F., Maestre Campos, M., Felipe Castaño, E., León del Barco, B., y Polo del Río, M. (2017). Análisis del rendimiento académico de los alumnos de educación secundaria obligatoria según las variables familiares. Educación XX1, 20 (1), 209-232.

Floyd, R.G., Evans,Y. \& Mc Grew,K.S.,(2003). Relations between measures of Catell Horn Carroll $(\mathrm{CHC})$ cognitive abilities and mathematics achievements across the school-age tears. Psychology in the Schools, 40 (2), 155-171.

Galibert,M.S., Aguerri, M.E., Pano, C.O., Luzzia, G.S., Attorresi, H.F.(2005). Análisis de item de analogías verbales mediante la aplicación de un modelo politómico de la teoría de respuesta al item. Revista Mexicana de psicología,22 (2), 419-431.

Geiser,S.(2016) Medición y evaluación para los procesos de admission de la educación superior. Hallazgos desde California. Pensamiento Educativo.Revista de Investigación Educacional Latinoamericana, 53 (1),1-18.

George, D. \& Mallery, P. (2003). SPSS for Windows step by step: A simple guide and reference. 11.0 update (4th ed.). Boston, MA: Allyn \& Bacon.

George, D., \& Mallery, M. (2011). SPSS for Windows step by step: A simple guide and reference 18.0 Update (11 ed.). Boston, MA: Allyn \& Bacon

Jiménez Morales, M., \& López-Zafra, E. (2009). Inteligencia emocional y rendimiento escolar: estado actual de la cuestión. Revista Latinoamericana de Psicología, 41 (1), 69-79.

Kuncel, Nathan R.,Hezlett, Sarah A.,Ones, Deniz, S. (2004) Academic Performance, Career potencial, Creativity and Job Performance; can one construct predict them all? Journal of Personality and Social Psychology, $86(1), 148-161$

Marchesi, A. y Martín, E. (1999). Calidad de la Enseñanza en tiempos de cambio. Madrid: Alianza Editorial.

Marrs H. (2011) Catell-Horn-Carroll Theory of Intelligence. In: Goldstein S., Naglieri J.A. (eds) Encyclopedia of Child Behavior and Development. Springer, Boston, MA

Pérez, E., \& Cupani, M., \& Ayllón, S. (2005). Predictores de rendimiento académico en la escuela media: habilidades, autoeficacia y rasgos de personalidad. Avaliação Psicológica, 4 (1), 1-11.

Pérez Sánchez, A., \& Castejón Costa, J. (1997). Factores que distinguen y explican el rendimiento académico diferencial de alumnos repetidores y alumnos cuya edad escolar coincide con su edad cronológica. Revista de Psicodidáctica, (4), 103-117.

Ribeiro (1998). Mudanzas no desepenho e na estructura das optisoes: contributos para o estado da diferenciazao cognitiva en jovens. Tesis de doutoramento. Braga. Universidade do Minho.

Ridgell, S. D., \& Lounsbury, J. W. (2004). Predicting academic success : general intelligence, "big 
five" personality traits, and work drive. College Student Journal, 38, (4), 607-618.

Robles,B.C., Vázquez González, E. (2014). La influencia de las competencias verbales en el ámbito escolar. International Journal of Developmental and Educational Psychology,6 (1), 351-362.

Sarmiento, J.A. y Ocampo,C.I. (2004). La orientación psicopedagógica desde la complejidad sistémica: un nuevo enfoque. Revista Española de Orientación y Psicopedagogía, 15 (2), 373-389.

Sebastián, A. (2003) (Coord.). Orientación profesional. Un proceso a lo largo de la vida. Madrid: Dykinson.

Sternberg, R. J., \& Berg, C. A. (1992). Intellectual development. Cambridge, UK: Cambridge University Press.

Yela, M.(1996) Los test y el análisis factorial. Psicothema, (8), 73-88 
